\title{
Role for TRPA1 receptor channels in trigeminal afferent activation and neuropeptide release from rat cranial dura mater
}

\author{
S Albrecht, AC Denner, M Eberhardt, R DeCol, KB Messlinger* \\ From The European Headache and Migraine Trust International Congress \\ London, UK. 20-23 September 2012
}

\section{Introduction}

TRPA1 receptor channels are activated by environmental irritants and by endogenous mediators released during inflammatory conditions (McMahon \& Wood 2006,). Activation of TRPA1 receptors causes CGRP release from trigeminal ganglion neurons and increases meningeal blood flow upon nasal stimulation (Kunkler et al. 2011), providing evidence that TRPA1 receptors may be involved in the generation of headaches.

\section{Objective}

To further examine the role of TRPA1 receptor activation in processes presumably associated with headache generation, we investigated the effects of the TRPA1 agonist acrolein on functions involved in meningeal nociception using four different rat models.

\section{Methods}

The discharge activity of single meningeal afferents innervating the dura mater was recorded in a hemisected cranial preparation. 2 . The activity of second order neurons in the spinal trigeminal nucleus with meningeal afferent input was recorded in anaesthetised animals. 3. In the hemisected cranial preparation the dura mater was superfused with synthetic interstitial fluid, and stimulated calcitonin generelated peptide (CGRP) release was measured using an ELISA. 4. Meningeal blood flow was monitored in the exposed dura mater of anaesthetised animals using laser Doppler flowmetry. In all preparations the dura mater was stimulated with the TRPA1 agonist acrolein (10-4 M).

\section{Results}

Acrolein did not elicit discharges in meningeal $A \delta$ - or $\mathrm{C}$-fibres in the hemisected cranial preparation and did not change the discharge activity of second order neurons with meningeal receptive fields in anaesthetized animals. In contrast, acrolein significantly stimulated CGRP release from the dura mater within $5 \mathrm{~min}$ and increased meningeal blood flow. Both responses were suppressed by the TRPA1 inhibitor HC030031.

\section{Conclusion}

TRPA1 channel activation causes neuropeptide release from meningeal afferents but does not generate propagated afferent information. Therefore an important role for peripheral TRPA1 receptors in headache generation appears unlikely.

\section{Published: 21 February 2013}

\section{References}

1. Kunkler PE, Ballard CJ, Oxford GS, Hurley JH: TRPA1 receptors mediate environmental irritant-induced meningeal vasodilatation. Pain 2011, 152:38-44.

2. McMahon SB, Wood JN: Increasingly irritable and close to tears: TRPA1 in inflammatory pain. Cell 2006, 124:1123-1125.

doi:10.1186/1129-2377-14-S1-P70

Cite this article as: Albrecht et al:: Role for TRPA1 receptor channels in trigeminal afferent activation and neuropeptide release from rat cranial dura mater. The Journal of Headache and Pain 2013 14(Suppl 1):P70. 